\section{Bewältigung von Pruritus}

\author{
Viele chronische Hauterkrankungen gehen mit einem belastenden, \\ die Lebensqualität einschränkenden Juckreiz einher. Niederländische \\ Dermatologen haben daher ein spezielles Bewältigungsprogamm \\ entwickelt und dessen Effektivität überprüft.
}

$\mathrm{D}$ as Programm „coping with itch“ der Universität Utrecht hatte sich schon in einer vorhergehenden Untersuchung bewährt - allerdings ohne Kontrollgruppe. Jetzt wurde es im Rahmen einer randomisierten Studie bei Patienten mit chronischem Pruritus überprüft. Die Studienteilnehmer wurden zwei Gruppen zugeordnet: 36 von ihnen erhielten eine ambulante Standardtherapie durch einen Dermatologen, die übrigen 29 nahmen zusätzlich an dem Programm teil. Das Programm bestand aus einer Schulung und einer kognitiv-behavioristischen Intervention in 45-minütigen Einzelstunden durch eine spezialisierte Krankenschwester. Zu Studienbeginn sowie nach drei und neun Wochen wurden anhand verschiedener Methoden der Juckreiz, das Kratzen, die Bewältigung des Juckreizes und die hautbezogene sowie die allgemeine psychosoziale Morbidität erfasst.
Die Patienten der Interventionsgruppe besuchten durchschnittlich 2,9 Einzelstunden - vorwiegend in den ersten drei Monaten. Tatsächlich litten diese Patienten nach drei Monaten im Vergleich zur Kontrollgruppe weniger unter Juckreiz und kratzten sich seltener, sta-

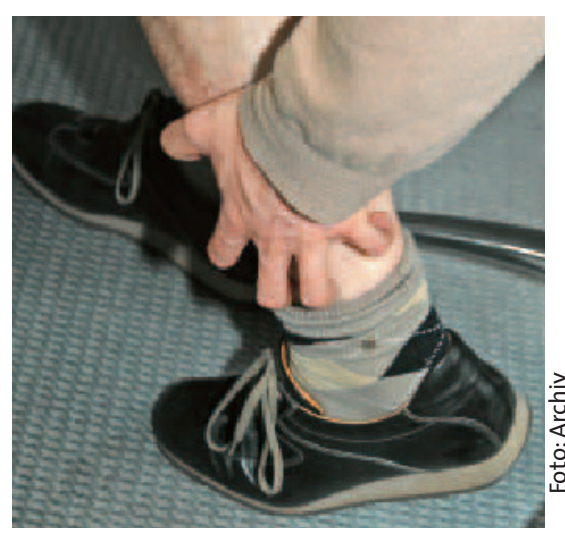

Quälender Juckreiz - hilft eine Bewältigungsprogramm? tistische Signifikanz wurde aber nicht erreicht $(p=0,07)$. Ein signifikanter Unterschied zugunsten des Programms zeigte sich lediglich bei der psychischen Bewältigung des Leidens. Nach neun Monaten waren alle Unterschiede in den beiden Gruppen allerdings wieder verschwunden. Da die Intervention überwiegend in den ersten drei Monaten stattfand, vermuten die Autoren, dass die Patienten für einen längerfristigen Erfolg zu kurz an dem Programm teilgenommen haben. Nur ein Effekt war während des gesamten Untersuchungszeitraumes vorhanden: Die Patienten der Interventionsgruppe suchten signifikant seltener einen Dermatologen auf im Vergleich zu den Patienten aus der Kontrollgruppe.

Fazit: Ein spezielles Bewältigungs-Programm kann Patienten mit chronischem Pruritus helfen, besser mit ihrem Leiden umzugehen, und reduziert im Trend die Kratzhäufigkeit. Der Effekt ist aber offenbar sehr begrenzt.

pe

van Os-Medendorp $\mathrm{H}$ et al. The nursing programme, Coping with itch': a promising intervention for patients with chronic pruritic skin diseases. J Clin Nurs 2007; 16: 1238-46

\title{
Patchtests im Patientenurteil
}

\author{
Patchtests sind in der Diagnostik der Kontaktallergie Standard. \\ Interessant ist die Frage, wie die Patienten diese Untersuchung \\ beurteilen und welche Konsequenzen sie aus dem Ergebnis für ihr \\ Alltagsleben ziehen.
}

D ermatologen der Mayo-Klinik im US-Bundesstaat Minnesota befragten per Post nachträglich $1.453 \mathrm{Kli}$ nikpatienten, bei denen zuvor Patchtests durchgeführt worden waren. Die Patienten sollten im Multiple-Choice-Verfahren Fragen zu ihrer Zufriedenheit mit dem Hauttest und zu den Konsequenzen der Diagnose beantworten. Zudem wurden die Studienteilnehmer gebeten, die Allergene zu benennen, auf die sie im Test positiv reagiert hatten. sogar als ,viel besser“. ein Allergen positiv gewesen. Von den positiv getesteten Allergenen wurde jedoch nicht einmal die Hälfte $(48,3 \%)$ von den Patienten korrekt wiedergegeben. $48,2 \%$ der Studienteilnehmer änderten aufgrund der Diagnose ihr Verhalten im Alltag. Von den Patienten, bei denen Allergien gegen Hautpflegemittel festgestellt worden waren, berücksichtigten über drei Viertel die entsprechenden Empfehlungen des Hautarztes. kam ausgefüllt wieder zurück. Insgesamt zeigten sich die Patienten mit der Durchführung des Tests zufrieden: $76,7 \%$ waren „einigermaßen zufrieden“ und $51,5 \%$,sehr zufrieden“. $58,3 \%$ berichteten über eine Verbesserung ihres Hautzustandes durch Berücksichtigung des Patchtestergebnisses, 32,1\% beurteilten den Zustand ihrer Haut dadurch

Bei 76,6\% der antwortenden Patienten war der Patchtest zumindest auf
Fazit: Die deutliche Mehrheit der Patienten beurteilt Patchtests als hilfreich. Allerdings ist nach wenigen Jahren mehr als die Hälfte der positiv getesteten Allergene bei den Patienten in Vergessenheit geraten.

Scalf LA et al. Patients' perceptions of the usefulness and outcome of patch testing. J Am Acad Dermatol 2007; 56: 928-32 